\title{
LETTERS
}

\section{Ensuring nondiscrimination in pandemic prioritization decisions}

We read with great interest the CMAJ commentary by Parhar and colleagues ${ }^{1}$ that described triage protocols in Alberta for extracorporeal life support (ECLS) resources. However, we are concerned that it raises questions by not explicitly mentioning which preexisting medical conditions would justify exclusion from resources.

Although we acknowledge that it is reasonable in a pandemic situation to limit access to some intensive resources like ECLS for patients with conditions that limit their chance of survival, these decisions need to be based on predicted patient outcome as judged by the best available evidence, using the most objective criteria possible. They must also be blind to societal factors such as race, religion or ability to pay. In the Canadian context, there is little reason to suspect that those societal factors would be considered by clinicians or systems.

The commentary did not specify which preexisting medical conditions would qualify for exclusion from ECLS. ${ }^{1}$ Other Canadian reports have gone further to propose the explicit exclusion of patients with preexisting cognitive impairments. ${ }^{2}$
We contend that comorbidities that do not influence expected probability to survive, notably preexisting cognitive or motor disability, should not be factored into triage decisions. If patients have healthy lungs, hearts and immune systems, there is no medical or ethical reason to bar them from benefitting from resources simply based on cognitive deficit.

There are no recognized legal standards that govern triage in Canada. As suggested by Parhar and colleagues, ${ }^{1}$ health care systems should develop policies to ensure each decision is made in a fair and transparent manner based on objective prognostic factors. ${ }^{3}$ Any decisions for a specific patient should not rest with an individual clinician.

People with disability, cognitive or otherwise, are human beings, entitled to the same legal and ethical protection as any other person who needs care in Canada.

\section{Matthew J. Weiss MD}

Pediatric intensive care physician, Division of Pediatric Intensive Care, Centre Mère-Enfant Soleil du CHU de Québec, Que.

\section{Franco Carnevale RN PhD}

Professor, Ingram School of Nursing, McGill University, Montréal, Que.

\section{Bram Rochwerg MD}

Assistant professor, Division of Critical Care, Department of Medicine, McMaster University, Hamilton, Ont.

\section{Simon Oczkowski MD}

Assistant professor, Division of Critical Care, Department of Medicine, McMaster University, Hamilton, Ont.

\section{Sonny Dhanani MD}

Pediatric intensive care unit chief, Children's Hospital of Eastern Ontario, Ottawa, Ont.

Cite as: CMAJ 2020 April 14;192:E421. doi: $10.1503 / \mathrm{cmaj} .75254$

\section{References}

1. Parhar KKS, Lequier L, Blackwood J, et al. Optimizing provision of extracorporeal life support during the COVID-19 pandemic: practical considerations for Canadian jurisdictions. CMAJ 2020; 192:E372-4

2. Christian MD, Hawryluck L, Wax RS, et al. Development of a triage protocol for critical care during an influenza pandemic. CMAJ 2006;175: 1377-81.

3. Upshur REG, Faith K, Gibson JL, et al. Stand on guard for thee: ethical considerations in preparedness planning for pandemic influenza. Toronto: University of Toronto, Joint Centre for Bioethics; 2005:1-29.

Competing interests: None declared. 